EPJ Web of Conferences 62, 04001 (2013)

DOI: $10.1051 /$ epjconf/20136204001

(C) Owned by the authors, published by EDP Sciences, 2013

\title{
Fission of actinides through quasimolecular shapes
}

\author{
Guy Royer ${ }^{1, \mathrm{a}}$, Hongfei Zhang ${ }^{2}$, Philippe Eudes ${ }^{1}$, Rachid Moustabchir $^{1}$, Damien Moreau ${ }^{1}$, \\ Muriel Jaffré ${ }^{1}$, Youssef Morabit ${ }^{1}$, and Benjamin Particelli ${ }^{1}$ \\ ${ }^{1}$ Laboratoire Subatech, UMR: IN2P3/CNRS-Université-Ecole des Mines, Nantes 44, France \\ ${ }^{2}$ School of Nuclear Science and Technology, Lanzhou University, Lanzhou 730000, China
}

\begin{abstract}
The potential energy of heavy nuclei has been calculated in the quasimolecular shape path from a generalized liquid drop model including the proximity energy, the charge and mass asymmetries and the microscopic corrections. The potential barriers are multiplehumped. The second maximum is the saddle-point. It corresponds to the transition from compact one-body shapes with a deep neck to two touching ellipsoids. The scission point lies at the end of an energy plateau well below the saddle-point and where the effects of the nuclear attractive forces between two separated fragments vanish. The energy on this plateau is the sum of the kinetic and excitation energies of the fragments. The shell and pairing corrections play an essential role to select the most probable fission path. The potential barrier heights agree with the experimental data and the theoretical half-lives follow the trend of the experimental values. A third peak and a shallow third minimum appear in asymmetric decay paths when one fragment is close to a double magic quasispherical nucleus, while the smaller one changes from oblate to prolate shapes.
\end{abstract}

\section{Introduction}

The analysis of the fission cross sections of actinides and of their ground and isomeric state properties indicates the existence of double-humped potential barriers. The fission probability and the angular distribution of the fragments suggest also the existence of hyperdeformed states in a deep third well in several Th and $\mathrm{U}$ isotopes [1,2]. It is also necessary to suppose fission barrier heights of $5-10 \mathrm{MeV}$ to explain the stability of superheavy elements of charge 112-118 [3, 4].

The fission shapes were firstly investigated by minimizing the sum of the surface and Coulomb energies using a development of the radius in Legendre polynomials. This leads to decay paths through very elongated shapes with shallow neck or no neck. Within a Generalized Liquid Drop Model (GLDM) taking into account the mass and charge asymmetries, the proximity energy, and the shell and pairing energies, it has been demonstrated that there is a degeneracy between the energy of quasimolecular shapes and the energy of elongated shapes with a shallow neck. Most of the binary and ternary fission, $\alpha$ and light nucleus emission and fusion properties have been also reproduced in this quasimolecular shape valley [5-9]. The purpose of this work is to investigate the actinide region remaining in this

\footnotetext{
ae-mail: royer@subatech.in2p3.fr
}

This is an Open Access article distributed under the terms of the Creative Commons Attribution License 2.0, which permits unrestricted use, distribution, and reproduction in any medium, provided the original work is properly cited. 


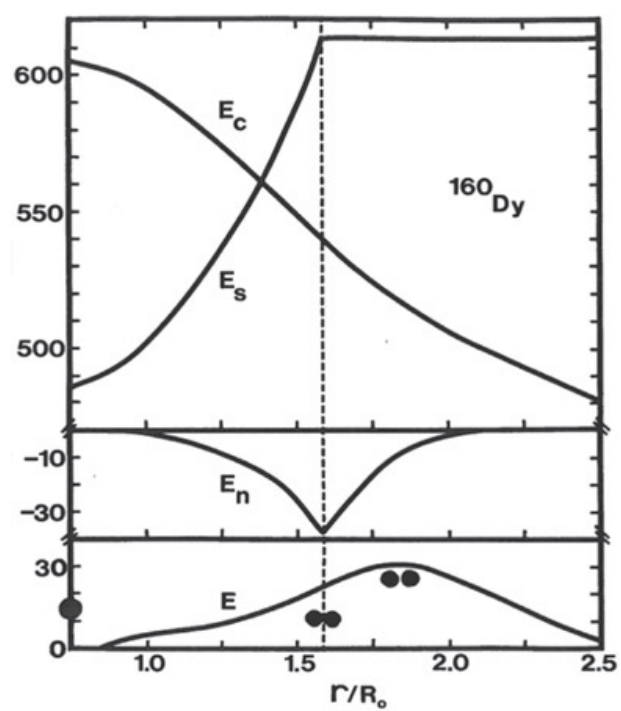

Figure 1. Contribution of the Coulomb, surface and nuclear proximity energies to the total deformation energy $\mathrm{E}$ of the ${ }^{160}$ Dy versus the distance between the mass centers. The dotted line indicates the contact point between spherical fragments.

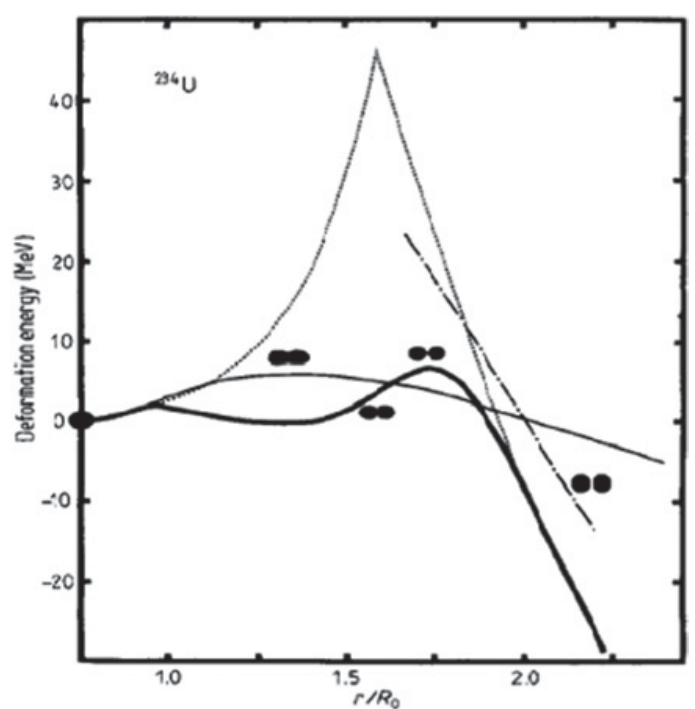

Figure 2. Fission barriers of ${ }^{234} \mathrm{U}$ : the dotted and thick full curves correspond to the potential for quasi-molecular shapes without and with proximity energy, the thin full curve gives the energy of elongated shapes while the chain curve is the energy of two separated oblate spheroids without proximity contribution.

peculiar fusionlike shape path, taking into account the ellipsoidal deformations of the fission fragments, studying with this GLDM all the possible mass and charge asymmetries, accounting for shell and pairing energies.

\section{Potential barriers}

Firstly, the macroscopic contributions to the deformation energy E derived from this GLDM energy are shown in Figure 1 for the ${ }^{160}$ Dy nucleus. The surface and proximity energies $E_{s}$ and $E_{n}$ change drastically at the contact point since the surface is constant after the separation and the nuclear attraction is greatest at the contact point. Nevertheless, the total energy varies gently even around the contact point. Moreover the barrier height corresponds to the fission barrier height [5]. In the Figure 2 the deformation energies of the ${ }^{234} \mathrm{U}$ nucleus corresponding to different shape sequences without and with proximity energy contribution are compared. The potential energy calculated using quasimolecular shapes without taking into account the proximity energy, the so-called Coulomb barrier, is very energetically unfavourable. On the contrary, when the proximity energy is included for the same shape sequence, the barrier height may be compared with the barrier height of the potential energy for usual elongated and little or not creviced shapes. Furthermore, a double-humped barrier appears even macroscopically for the compact and necked shapes. This shows clearly that the comparison between the two shape sequences must be re-examined when the additional proximity energy term is taken into account. The two spheroid shapes would be also highly competitive with regard to the usual elongated shapes if the proximity energy was included.

The picture of the Businaro-Gallone point assuming that, macroscopically, asymmetric fission is favoured for light systems and symmetric fission for heavy nuclei is also observed in the quasimolecular 

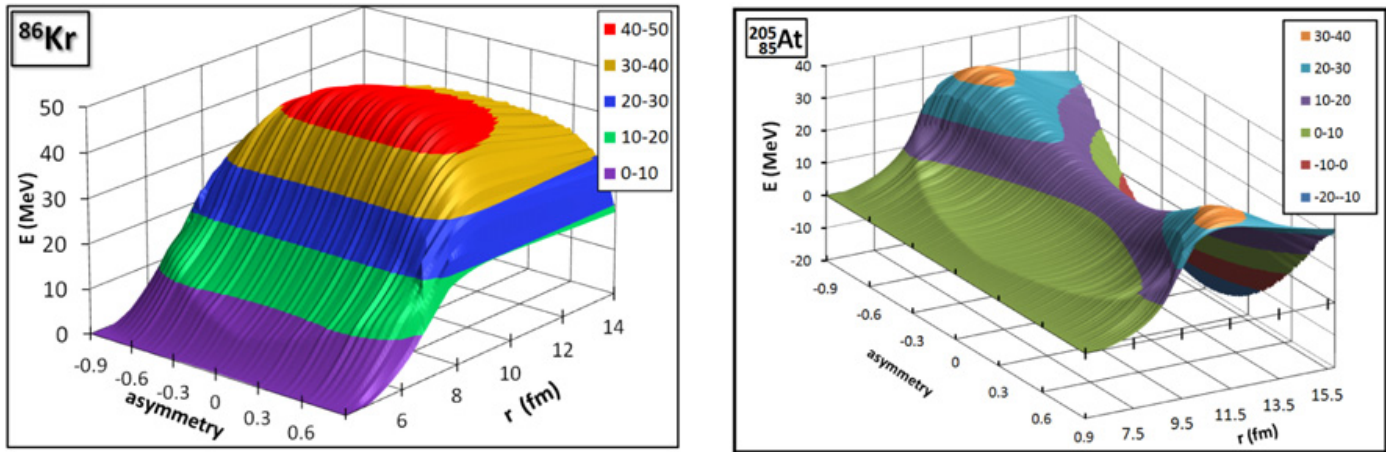

Figure 3. Macroscopic fission barriers as functions of the decay asymmetry $\left(A_{1}-A_{2}\right) /\left(A_{1}+A_{2}\right)$ and the distance between the mass centers $\mathrm{r}$ for the two ${ }^{86} \mathrm{Kr}$ and ${ }^{205}$ At nuclei.

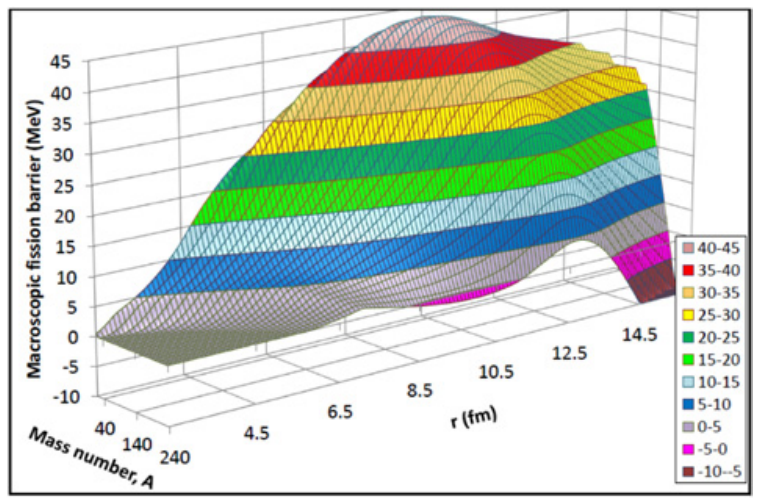

Figure 4. Macroscopic barriers of the symmetric fission in the beta-stability valley and in the quasimolecular shape valleys.

shape path (see Fig. 3). More generally, it has been proved that the potential barrier heights in this valley correspond precisely to the fission barrier heights [5-9].

The dependence of the potential barriers on the mass of the decaying nucleus is displayed in Figure 4. With increasing mass appear macroscopically a plateau and a second external relative minimum and internal peak due to the proximity energy and then the possibility of isomeric states.

In Figure 5 the shell and pairing energies have been introduced as well as the ellipsoidal deformations of the fragments to calculate the different exit channels of ${ }^{240} \mathrm{Pu}$. Multiple-humped potential barriers appear. The heights of the two peaks are displayed on Figure 6. The height of the first peak is almost constant since it depends on the shell effects around the ground state. The shell effects and the proximity energies flatten the potential energy surface and generate a shallow second minimum. The second maximum, the saddle-point, corresponds to the transition from compact and creviced onebody shapes to two touching oblate ellipsoids. The height of this second peak generally increases with the asymmetry but the shell and pairing energies lead to strong distortions from this general behaviour. The barrier for the most probable exit channel is also shown on Figure 7. The scission point corresponds to the vanishing of the proximity energy and the effective separation of the fragments. It occurs on an energy plateau corresponding to the fragment kinetic energy plus an excitation energy. The evolution of the shell effects and shapes of the fragments play a main role during the descent from the saddle-point to the scission point (see also [10]). 


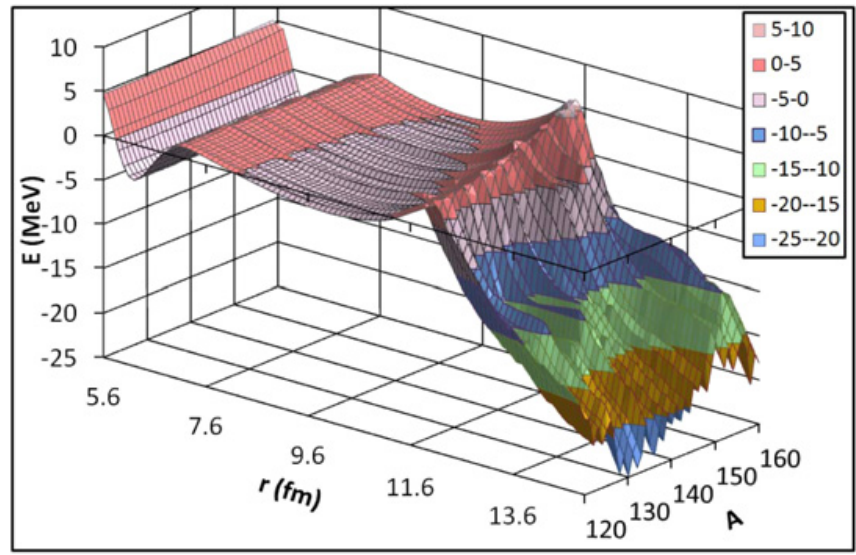

Figure 5. Multiple-humped microscopic barriers for ${ }^{240} \mathrm{Pu}$ as a function of the heaviest fragment mass.

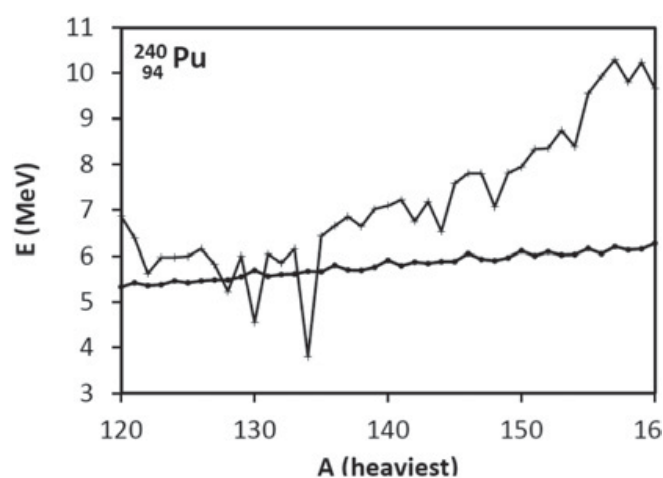

Figure 6. Heights of the inner and outer peaks of the fission barrier versus the mass of the heaviest fragment.

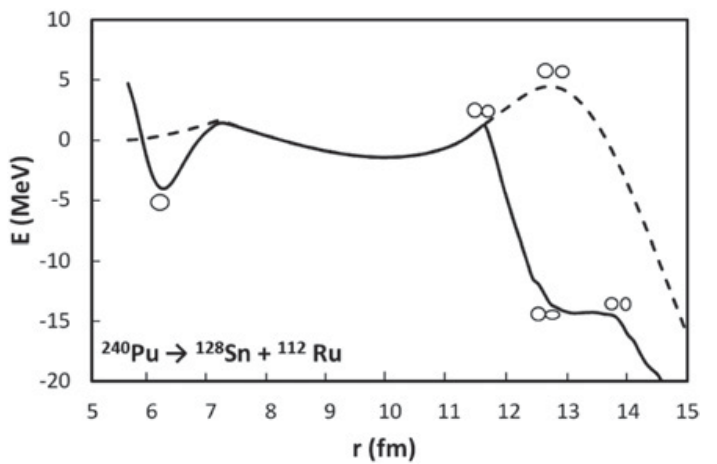

Figure 7. Asymmetric fission barrier of a ${ }^{240} \mathrm{Pu}$ nucleus emitting a magic nucleus ${ }^{128} \mathrm{Sn}$. The dashed curve corresponds to the macroscopic energy within the twosphere approximation for the two-body shapes while the solid line includes the microscopic corrections and the ellipsoidal deformations of the fragments.

Shallow third minimum and peak appear in specific asymmetric exit channels where one fragment is close to a double magic quasi-spherical nucleus while the other one evolves from oblate to prolate shapes. Then the third peak corresponds to two separated spheres and the maximum of the shell effects. Such triple-humped barriers do not appear in symmetric exit channels.

The heights of the two peaks of the potential barriers roughly agree with the experimental data [9].

\section{Fission half-lives}

Within this asymmetric fission model the decay constant is simply the product of the assault frequency by the barrier penetrability. For shapes near the ground state the selected inertia is largely above the irrotational flow value since a large amount of internal reorganization occurs at level crossings [9]. For highly deformed shapes the reduced mass is reached asymptotically.

The experimental spontaneous fission half-lives and theoretical predictions are compared in Table 1. There is a rough agreement with most of the experimental data on 26 orders of magnitude for the 
Table 1. Comparison between experimental and theoretical spontaneous fission half-lives.

\begin{tabular}{|c|c|c|c|c|}
\hline Nucleus & $\mathrm{T}_{1 / 2, \exp }(\mathrm{s})$ & $\mathrm{T}_{1 / 2}(\mathrm{~s})[9, \mathrm{Z}=114]$ & $\mathrm{T}_{1 / 2}(\mathrm{~s})[9, \mathrm{Z}=116]$ & $\mathrm{T}_{1 / 2,(\mathrm{~s})[11]}$ \\
\hline${ }^{234} \mathrm{U}$ & $4.7 \times 10^{23}$ & $4.6 \times 10^{19}$ & $1.1 \times 10^{21}$ & $2.9 \times 10^{22}$ \\
\hline${ }^{236} \mathrm{U}$ & $7.8 \times 10^{23}$ & $1.7 \times 10^{22}$ & $5.4 \times 10^{23}$ & $1.7 \times 10^{22}$ \\
\hline${ }^{238} \mathrm{U}$ & $2.6 \times 10^{23}$ & $5.2 \times 10^{23}$ & $1.1 \times 10^{25}$ & $1.1 \times 10^{21}$ \\
\hline${ }^{239} \mathrm{Pu}$ & $2.5 \times 10^{23}$ & $9.9 \times 10^{22}$ & $4.1 \times 10^{24}$ & $2.7 \times 10^{22}$ \\
\hline${ }^{240} \mathrm{Pu}$ & $3.7 \times 10^{18}$ & $2.3 \times 10^{20}$ & $1.2 \times 10^{22}$ & $5.1 \times 10^{22}$ \\
\hline${ }^{243} \mathrm{Am}$ & $6.3 \times 10^{21}$ & $3.6 \times 10^{22}$ & $2.0 \times 10^{24}$ & $6.9 \times 10^{20}$ \\
\hline${ }^{243} \mathrm{Cm}$ & $1.7 \times 10^{19}$ & $2.3 \times 10^{16}$ & $6.0 \times 10^{18}$ & $4.4 \times 10^{20}$ \\
\hline${ }^{245} \mathrm{Cm}$ & $4.4 \times 10^{19}$ & $2.0 \times 10^{20}$ & $2.2 \times 10^{22}$ & $8.6 \times 10^{20}$ \\
\hline${ }^{248} \mathrm{Cm}$ & $1.3 \times 10^{14}$ & $1.9 \times 10^{18}$ & $2.3 \times 10^{20}$ & $4.6 \times 10^{18}$ \\
\hline${ }^{249} \mathrm{Bk}$ & $6.1 \times 10^{16}$ & $1.1 \times 10^{16}$ & $6.2 \times 10^{16}$ & $2.1 \times 10^{16}$ \\
\hline${ }^{250} \mathrm{Cf}$ & $5.2 \times 10^{11}$ & $4.2 \times 10^{11}$ & $1.2 \times 10^{15}$ & $4.1 \times 10^{16}$ \\
\hline${ }^{253}$ Es & $2.0 \times 10^{13}$ & $3.7 \times 10^{9}$ & $3.7 \times 10^{9}$ & $2.6 \times 10^{12}$ \\
\hline${ }^{255}$ Es & $8.4 \times 10^{10}$ & $5.5 \times 10^{6}$ & $3.5 \times 10^{9}$ & $2.3 \times 10^{8}$ \\
\hline${ }^{250} \mathrm{Fm}$ & $2.6 \times 10^{7}$ & $1.8 \times 10^{3}$ & $4.3 \times 10^{5}$ & $4.5 \times 10^{7}$ \\
\hline${ }^{254} \mathrm{Fm}$ & $1.9 \times 10^{7}$ & $1.9 \times 10^{4}$ & $5.6 \times 10^{6}$ & $5.2 \times 10^{7}$ \\
\hline${ }^{255} \mathrm{Md}$ & $1.1 \times 10^{6}$ & $1.8 \times 10^{2}$ & $4.1 \times 10^{4}$ & $8.4 \times 10^{5}$ \\
\hline${ }^{257} \mathrm{Md}$ & $2.0 \times 10^{6}$ & $1.1 \times 10^{3}$ & $2.7 \times 10^{4}$ & $1.8 \times 10^{5}$ \\
\hline${ }^{259} \mathrm{Md}$ & $5.8 \times 10^{3}$ & $7.7 \times 10^{-1}$ & $1.3 \times 10^{3}$ & $2.9 \times 10^{3}$ \\
\hline${ }^{252} \mathrm{No}$ & $1.2 \times 10^{1}$ & $2.5 \times 10^{-1}$ & $4.3 \times 10^{1}$ & $2.2 \times 10^{4}$ \\
\hline${ }^{254} \mathrm{No}$ & $3.0 \times 10^{4}$ & $1.1 \times 10^{0}$ & $1.9 \times 10^{2}$ & $5.1 \times 10^{4}$ \\
\hline${ }^{256} \mathrm{No}$ & $1.1 \times 10^{2}$ & $1.9 \times 10^{0}$ & $3.7 \times 10^{2}$ & $2.1 \times 10^{4}$ \\
\hline${ }^{252} \mathrm{Lr}$ & $3.6 \times 10^{1}$ & $1.6 \times 10^{-3}$ & $2.6 \times 10^{-1}$ & $2.0 \times 10^{2}$ \\
\hline${ }^{256} \mathrm{Lr}$ & $9.0 \times 10^{5}$ & $3.6 \times 10^{-2}$ & $6.7 \times 10^{0}$ & $3.3 \times 10^{2}$ \\
\hline${ }^{259} \mathrm{Lr}$ & $5.8 \times 10^{3}$ & $1.3 \times 10^{-3}$ & $1.3 \times 10^{0}$ & $3.4 \times 10^{2}$ \\
\hline${ }^{256} \mathrm{Rf}$ & $6.4 \times 10^{-3}$ & $3.5 \times 10^{-4}$ & $5.6 \times 10^{-2}$ & $4.0 \times 10^{1}$ \\
\hline${ }^{258} \mathrm{Rf}$ & $9.4 \times 10^{-2}$ & $8.2 \times 10^{-4}$ & $1.6 \times 10^{-1}$ & $4.2 \times 10^{1}$ \\
\hline${ }^{260} \mathrm{Rf}$ & $5.1 \times 10^{-2}$ & $2.8 \times 10^{-4}$ & $4.7 \times 10^{-2}$ & $3.6 \times 10^{0}$ \\
\hline${ }^{255} \mathrm{Db}$ & $8.0 \times 10^{-1}$ & $2.5 \times 10^{-6}$ & $3.6 \times 10^{-4}$ & $3.5 \times 10^{-1}$ \\
\hline${ }^{258} \mathrm{Sg}$ & $5.2 \times 10^{-3}$ & $1.1 \times 10^{-6}$ & $2.2 \times 10^{-4}$ & $2.9 \times 10^{-2}$ \\
\hline${ }^{262} \mathrm{Sg}$ & $7.0 \times 10^{-3}$ & $2.9 \times 10^{-7}$ & $5.2 \times 10^{-5}$ & $3.9 \times 10^{-5}$ \\
\hline${ }^{264} \mathrm{Hs}$ & $1.6 \times 10^{-3}$ & $2.1 \times 10^{-10}$ & $3.5 \times 10^{-8}$ & $5.1 \times 10^{-8}$ \\
\hline${ }^{286} \mathrm{Fl}$ & $1.3 \times 10^{-1}$ & $8.0 \times 10^{3}$ & $7.2 \times 10^{-2}$ & $6.9 \times 10^{-2}$ \\
\hline
\end{tabular}

actinides. The same approach has been applied to superheavy elements. The GLDM leads to too low half-life values assuming that $\mathrm{Z}=114$ is the next proton magic number. A better agreement is obtained selecting $Z=116$. Finally the last column of table 1 gives the values obtained with an improved version of the GLDM [11] which uses slightly different parameters and calculates the shell effects from a Woods-Saxon potential, the pairing energy being shape dependent.

\section{Summary and conclusion}

Potential barriers of actinides in the quasimolecular shape path have been studied within a generalized liquid drop model including the nuclear proximity energy and microscopic corrections.

Double-humped potential barriers and large deformed minima lodging possibly isomeric states appear. The external saddle-point corresponds to the transition from one-body creviced shapes to two touching ellipsoids. The scission point, where the effects of the nuclear attractive forces between the fragments vanish, lies at the end of an energy plateau below the saddle-point. The energy on this plateau corresponds to the fragment kinetic energy plus an excitation energy. The barrier heights roughly agree with the experimental data. The shell and pairing effects play a main role to decide the most probable decay path. The predicted half-lives follow most of the experimental data. A shallow third minimum 
and a third peak appear in specific asymmetric exit channels where one fragment is close to a double magic quasispherical nucleus, while the other one evolves from oblate to prolate shapes.

\section{References}

[1] J. Blons, C. Mazur, D. Paya, M. Ribrag, H. Weigmann, Nucl. Phys. A 414, 1 (1984)

[2] A. Krasznahorkay et al, Phys. Lett. B 461, 15 (1999)

[3] P. Armbruster, Eur. Phys. J. A 37, 159 (2008)

[4] V. Zagrebaev, W. Greiner, Phys. Rev. C 78, 034610 (2008)

[5] G. Royer, B. Remaud, J. Phys. G 10, 1541 (1984)

[6] G. Royer, B. Remaud, Nucl. Phys. A 444, 477 (1985)

[7] G. Royer, J. Phys. G 26, 1149 (2000)

[8] G. Royer, J. Gaudillot, Phys. Rev. C 84, 044602 (2011)

[9] G. Royer, M. Jaffré, D. Moreau, Phys. Rev. C 86, 044326 (2012)

[10] S. Panebianco, J.L. Sida, H. Goutte, J.F. Lemaître, N. Dubray, S. Hilaire, Phys. Rev. C 86, 064601 (2012)

[11] X.J. Bao, H.F. Zhang, G. Royer, J.Q. Li, Nucl. Phys. A 906, 1 (2013) 